\title{
Implementation of a Collision Avoidance System To Assist Safe Driving Based on Data Fusion in Vehicular Networks
}

\author{
Haeyoung Lee ${ }^{1}$, Jihoon Yang ${ }^{1}$, and Klaus Moessner ${ }^{2}$ \\ ${ }^{1} 5 \mathrm{GIC}$, Institute for Communcation Systems (ICS), University of Surrey, U.K. \\ ${ }^{2}$ Professorship of Communications Engineering, Faculty of Electronics and information Technology, \\ University of Technology Chemnitz, Germany, and \\ 5GIC, Institute for Communication Systems, University of Surrey, UK. \\ Email: \{Haeyoung.Lee, J.Yang, K.Moessner\}@ surrey.ac.uk
}

\begin{abstract}
With development of 5G and Beyond communication technologies and the recent achievements in autonomous driving, technical solutions to improve road safety have attracted great attention. In this paper, we present a collision avoidance system implemented using a 1/10 scale vehicle, as a research platform for autonomous driving connected a vehicular network. While the collision avoidance system exploits data fusion to make decisions relevant to predicting potential collision events, the effectiveness of the fusion of data obtained from in-vehicle sensors and vehicular communication is evaluated within a testbed environment.
\end{abstract}

Index Terms-Vehicular networks, V2X, Internet of vehicles, collision avoidance, autonomous driving, ADAS

\section{INTRODUCTION}

$5 \mathrm{G}$ and Beyond (5G/B5G) is expected to promise bandwidth in excess of $100 \mathrm{Mbps}$ at latencies of less than $1 \mathrm{~ms}$, as well as to provide connectivity to very large number of devices [1]. Based on their capability, these technologies are envisaged to support three types of services: enhanced Mobile Broadband (eMBB), massive Machine Type Communications (mMTC), and ultra Reliable Low Latency Communications (uRLLC), serving a vast range of verticals such as manufacturing [2], agriculture, healthcare, transportation, etc [3]. Especially, emerging 5G/B5G based vehicle-to-everything (V2X) communication, which encompasses all types of communications involving vehicles, including vehicle-to-vehicle (V2V), vehicleto-infrastructure (V2I), and vehicle-to-pedestrian (V2P) communication modes, has attracted great attention in both academia and industries.

The recent achievements in autonomous driving and the demands for efficient traffic management connected to smart city applications have driven the development of intelligent transport systems (ITS) [4]. One of the significant areas of interest in today's transport vertical is the development of technical solutions aimed at improving road safety. The evolution of vehicular networks is envisaged to aid to improve driving safety including avoiding accidents while it will realise the Internet of Vehicles (IoV) which implements the Internet of Things (IoT) in vehicular environments [5].
Many techniques have been considered to reduce vehicle collisions by providing the driver with a warning prior to a potential collision. While they are a part of advanced driver assistance systems (ADAS), on-board sensors such as Radio Detection and Ranging (RADAR), Light Detection and Ranging (LiDAR), and optical sensors (cameras) are frequently used to monitor the vehicle surroundings and predict collisions [6], [7]. While limitations in each technology affect their suitability for meeting all mission-critical requirements of a collision avoidance function, the fusion of data generated by multiple sensors is widely adopted. Fusion refers to overcome the functional and environmental limitations of each type of sensor by generating an estimate of the state of each surrounding object with higher accuracy [8]. However, this data fusion mechanism has limitations in terms of reliability. As most sensor technologies can only detect objects in the line of sight (LOS) area, additional accident-prevention capabilities are required to also cover non-line of sight (NLOS) [9]. In order to further improve road safety, it is paramount to obtain information on objects in NLOS regions as well as to improve the detection range and reliability. Regarding this issue, V2X communication is recognised as a way to enable vehicles to exchange their own information with surroundings. Integration of V2X communication and in-vehicle sensor data fusion are widely studied in literature; In [8], for vehicle trajectory prediction and collision warning purpose, data fusion is proposed and evaluated via computer simulation. Radar and V2X communication fusion is suggested in [10] to enhance the perception range and reduce errors in position and velocity estimations. In these works, their proposed approach is tested in computer simulation.

In this paper, we present our collision avoidance system using a 1/10 scale vehicle platform. Possible collisions are detected through fusion of data from in-vehicle sensors including LiDAR and cameras with the support of V2I communication. While the collision avoidance function is implemented and employed in the vehicle platform as a first step, our work is extendable for a wide range of autonomous vehicle platforms 
to investigate algorithms of useful techniques (e.g., lane detection, V2V communication).

The reminder of the paper is organised as follows. In Section II, the high-level architecture of our system is described including characteristics of adopted in-vehicle sensors and V2X communication. The implementation method for the collision avoidance system with the vehicular platform is explained in Section III. The considered experimental scenario and performance validation are elaborated in Section IV to show the effectiveness of our proposed system. Finally, this paper is concluded in Section V.

\section{SySTEM OVERVIEW}

For the reliable and accurate collision avoidance function, as aforementioned, integrating in-vehicle sensor data and V2X communication is essential. It will enable to analyze the current situations more accurately and to generate an appropriate warning to the driver prior to a possible collision event. In this section, the high-level architecture of our collision avoidance system, characteristics of multiple types of sensors and V2X communication are presented.

\section{A. High-level Architecture of the collision avoidance system}

The architecture of our system is illustrated in Fig. 1. It comprises of three layers: Perception, Network, and Computing Control layer.

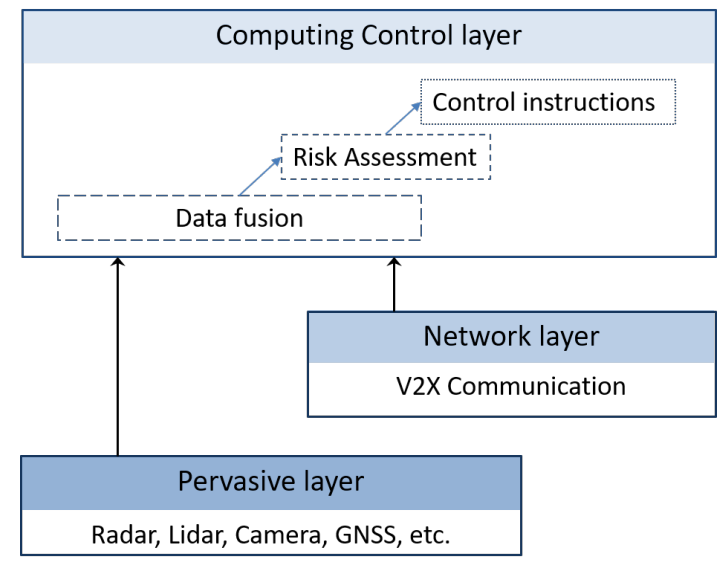

Fig. 1. The high-level architecture of our system

The first phase of the system is associated to perception. In the Perception layer, all types of sensors gather the environmental data inside/outside the vehicles to detect any specific events [5]. Multiple types of sensors including video cameras, RADARs, LiDARs, Global Positioning System (GPS) receivers, and other types of sensors such as Radio Frequency Identification (RFID) can be exploited to allow the sensing and perception of human, vehicles, and other things in the surrounding space.

The Network layer supports communication between the host vehicle and smart devices outside the vehicle. Various types of V2X connections (i.e., V2I, V2V, V2P, etc.) are supported by using IEEE 802.11p and cellular based V2X
(C-V2X) [11]. This layer is in charge of inter-connection between the host vehicle and other entities in the surroundings by providing the seamless heterogeneous network access. For V2X communication, more details are elaborated in Sec. II-C.

Lastly, in the Computing Control layer [12], collected data from Perception and Network layers can be stored and analysed with statistics tools. Based on data fusion, risk assessment can be undertaken. Along with decision making in risk assessment, another important function of this layer is to execute control instructions. For instance, data fusion processing with data obtained by RADARs, LiDARs, cameras and V2X communication is carried out. Then, based on the results from data fusion, in the risk assessment step, possible vehicle collisions can be detected and the distance between the vehicle and the target can be calculated. At the same time, instruction on the host vehicle brake system will be executed.

\section{B. Automotive Sensors for Information Gathering}

In our system, three types of in-vehicle sensors are considered; LiDAR, camera and Inertial Measurement Unit (IMU). The employed LiDAR covers up to $100 \mathrm{~m}$ range and the camera can cover up to $20 \mathrm{~m}$. While the two sensors are to monitor surroundings of the vehicle, the IMU is used for the vehicle odometry. The detailed specification of those sensors are listed in Table I.

TABLE I

HARDWARE SPECIFICATION OF THE VEHICLE PlatForm

\begin{tabular}{|l|l|}
\hline Type & Main specifications \\
\hline \hline 3D LiDAR & Velodyne VLP-16 \\
& Measurement Range: Up to $100 \mathrm{~m}$ \\
& Accuracy: \pm 3cm (Typical) \\
& Field of View (Vertical): $30^{\circ}\left[-15.0^{\circ},+15.0^{\circ}\right]$ \\
& Angular Resolution (Vertical): $2.0^{\circ}$ \\
& Field of View (Horizontal): $360^{\circ}$ \\
& Angular Resolution (Horizontal/Azimuth): $0.1^{\circ}-0.4^{\circ}$ \\
& Rotation Rate: 5-20 Hz \\
\hline Camera & ZED Stereo, 3D Sensing: Up to 20m \\
& Video Output: $2.2 \mathrm{~K}(15 \mathrm{FPS}, 4416 \times 1242)$, \\
& 1080p (340 FPS, 3840x1080), 720p (60 FPS. 2560x720), \\
& WVGA (100 FPS 1344x376) \\
& Field of View: $90^{\circ}(\mathrm{H}) \times 60^{\circ}(\mathrm{V}) \times 100^{\circ}(\mathrm{D})$ max. \\
& Depth Range: $0.3-25 \mathrm{~m}$ \\
& Depth FPS: Up to 100Hz \\
\hline IMU & SparkFun 9FoF Razor \\
& MPU-9250 9DoF (Degrees of Freedom) \\
& SAMD21 microprocessor \\
\hline
\end{tabular}

\section{V2X Communications}

$\mathrm{V} 2 \mathrm{X}$ communication can contribute to increasing the accuracy of object detection in collision avoidance. It can enable the exchange of data between vehicles and entities outside the vehicle. The interaction between multiple connected entities consists of information exchange through the adoption of suitable communication protocols, such as IEEE 802.11p [13] and C-V2X standards [14]. Fig. 2 [15] illustrates possible $\mathrm{V} 2 \mathrm{X}$ communication modes. In this paper, we focus on the V2I mode. V2I enables moving vehicles to communicate with road side units (RSUs) such as traffic lights, cameras, lane 


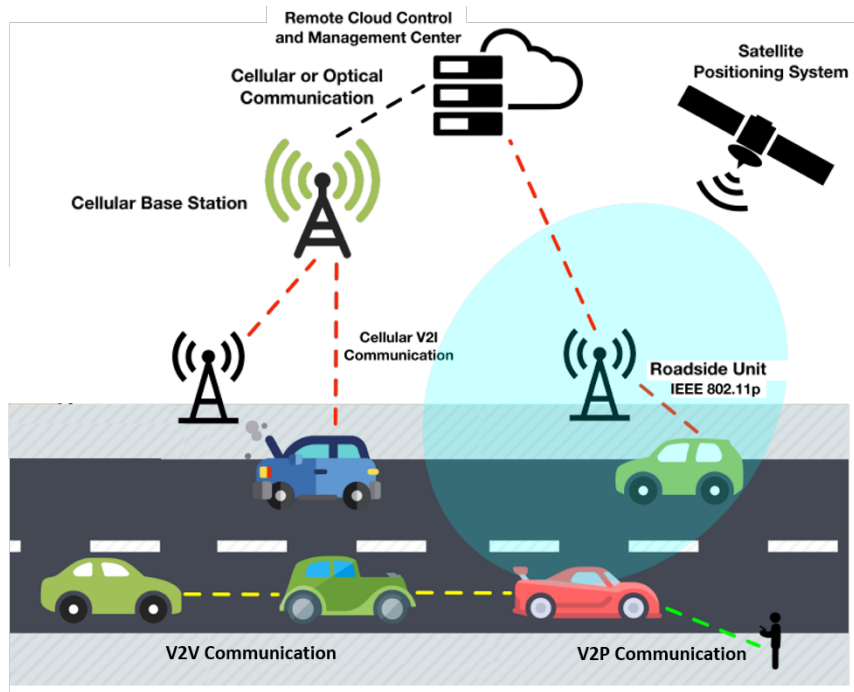

Fig. 2. V2X Communication including various types of communication

markings, street lamps, and signage. RSUs can act as standalone units or as relay nodes that provide safety messages and traffic updates. In our system, the RSU is assumed to inform the vehicle about detected suspicious entities or about road status so that the vehicle can utilise the information for the collision avoidance function.

\section{IMPLEMENTATION}

In this section, we present in detail the implementation of our system. The hardware components of both the vehicle platform and the RSU are explained as well as the software architecture and implementation.

\section{A. Hardware of the Vehicle Platform}

The vehicle platform including all on board components is shown in Fig. 3. The vehicle's body is a $1 / 10$ scale race car chassis. At the bottom part, there are two motors and battery packs. While the brushless DC motor is to provide power to all wheels, the servo motor controls the Ackermann steering angle [16]. The motors are controlled by a Vedder's

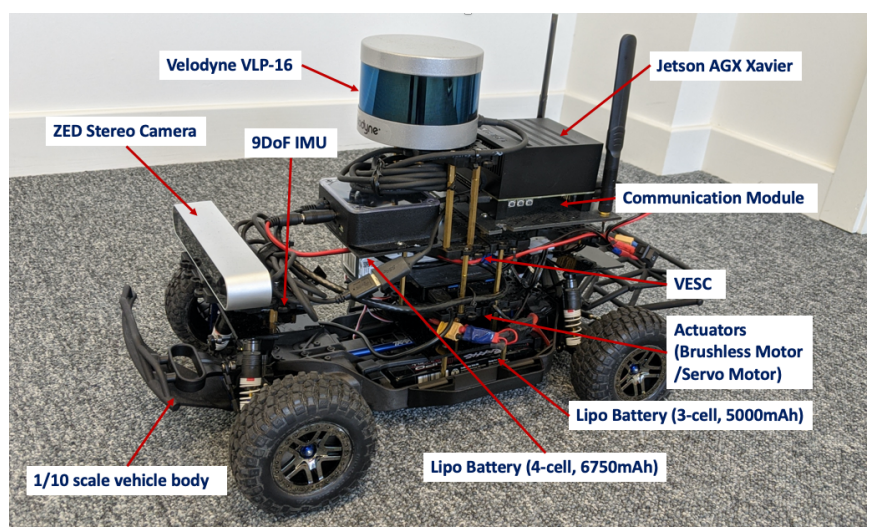

Fig. 3. The vehicle platform adopting various types of sensors
TABLE II

HARDWARE SPECIFICATIONS OF THE VEHICLE PlATFORM

\begin{tabular}{|c|c|c|}
\hline Type & Model & Detail specifications \\
\hline $\begin{array}{l}\text { Vehicle } \\
\text { body }\end{array}$ & $\begin{array}{l}\text { TRAXXAS } \\
\text { Slash } \\
4 \times 4 \\
\text { Ultimate }\end{array}$ & $\begin{array}{l}\text { 4WD (Four-Wheel Drive), } 1 / 10 \text { Scale } \\
\text { Size: } 568 \mathrm{~mm}(\mathrm{~L}) \text { x } 296 \mathrm{~mm}(\mathrm{~W}) \\
\text { Tire Diameter (front/rear): } 109.5 \mathrm{~mm} \\
\text { Wheelbase: } 324 \mathrm{~mm}\end{array}$ \\
\hline \multirow[t]{2}{*}{ Actuators } & Wheels & $\begin{array}{llll}\text { (TRX3351R) } & \text { Brushless } & \text { Motor } 3500 \mathrm{kV} \text {, } \\
\text { 4pole }\end{array}$ \\
\hline & $\begin{array}{l}\text { Steering } \\
\text { Angle }\end{array}$ & $\begin{array}{l}\text { (2075R) Servo Motor, Digital high-speed, } \\
\text { Metal gear (ball bearing) }\end{array}$ \\
\hline $\begin{array}{l}\text { Motor } \\
\text { Control }\end{array}$ & VESC & $\begin{array}{l}\text { Current, Speed, Duty-cycle, Field-Oriented } \\
\text { control available }\end{array}$ \\
\hline \multirow[t]{2}{*}{ Battery } & $\begin{array}{l}\text { TRAXXAS } \\
\text { Lipo }\end{array}$ & 3-cell, 11.1V, 25C, 5000mAh \\
\hline & $\begin{array}{l}\text { TATTU } \\
\text { Lipo }\end{array}$ & 4-cell, $14.8 \mathrm{~V}, 25 \mathrm{C}, 6750 \mathrm{mAh}$ \\
\hline $\begin{array}{l}\text { Communi } \\
\text {-cation }\end{array}$ & $\begin{array}{l}\text { Intel Dual } \\
\text { Bank }\end{array}$ & $\begin{array}{l}\text { (AC } 8265 \mathrm{NGW}) 2 \times 211 \mathrm{ac} \text { Wi-Fi delivering } \\
\text { up to } 867 \mathrm{Mbps}\end{array}$ \\
\hline Computer & $\begin{array}{l}\text { NVIDIA } \\
\text { Jetson } \\
\text { AGX } \\
\text { Xavier }\end{array}$ & $\begin{array}{l}\text { GPU: 512-core Volta } \\
\text { CPU: 8-core ARM v8,2 64-bit, 8MB L2 + } \\
\text { 4MB L3 } \\
\text { Memory:32GB 256-Bit LPDDR4x 137GB/s } \\
\text { Vision Accelerator: 7W VLIW Processor } \\
\text { Encoder/Decoder: [2x] 4Kp60, HEVC/[2x] } \\
\text { 4Kp60, 12-Bit Support }\end{array}$ \\
\hline
\end{tabular}

Electronic Speed Controller (VESC). For the purpose of data collection, as described in Sec. II-B, three sensors including a camera, LiDAR, and IMU, are deloyed. IEEE 802.11ac is selected for wireless communication, this will replaced by a IEEE 802.11p module in the future. Since the vehicle platform needs the computing capability like an edge-computing device, the platform is equipped with an NVIDIA Jetson AGX Xavier. The specifications of each component are listed in Table II.

\section{B. Hardware of the RSU}

The RSU is designed to implement a real world scenario to support communication between the moving vehicle and the RSU. The RSU illustrated in Fig. 4 consists of three parts, camera, communication module, and a computing module. While the same communication module described in Table II is used, the specifications of the camera and computing device are listed in Table III.

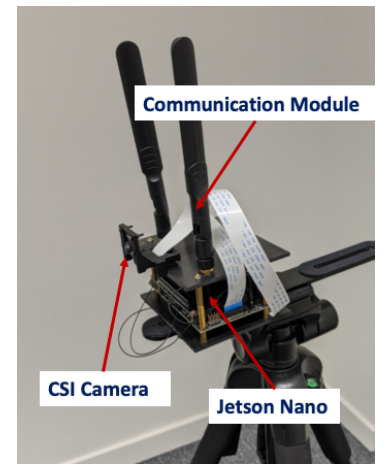

Fig. 4. The model of RSU adopting the camera sensor 
TABLE III

CAMERA SPECIFICATIONS OF THE RSU MODULE

\begin{tabular}{|l|l|l|}
\hline Type & Model & Detail specifications \\
\hline \hline Sensors & CSI Camera & Sony IMX 219 PQ CMOS image sensor in \\
& & a fixed-focus module \\
& & 8-megapixel, 3280 x 2464 \\
& & 1080p: 30fps, 720p: 60fps \\
\hline Computer & NVIDIA & GPU: 128-core Maxwell \\
& Jetson & CPU: Quad-core ARM A57@ 1.43GHz \\
& Nano & Memory: 4GB 256-Bit LPDDR4 25.6GB/s \\
& & Video Encode: 4K@30, 4x1080p@30, \\
& $9 x 720 p @ 30 \quad$ (H.264/H.265) 4K@60, \\
& & 2x4K@30, 8x1080p@30, 18x720p@30 \\
& & (H.264/H.265) \\
\hline
\end{tabular}

\section{Software}

The functional part of the software follows the high-level structure of the system described in Sec. II-A. By using a modular software design, we implement the hardware dependent parts as separate modules, to ensure extendability and portability. That is, algorithms tested in our testbed can be easily deployed in other hardware systems (e.g., 1/5 scale vehicle).

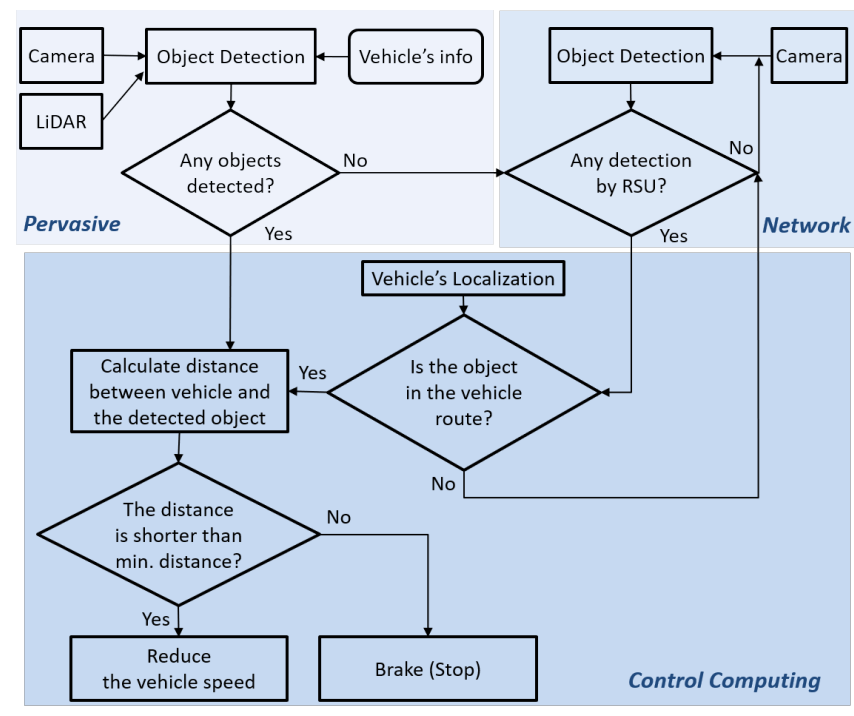

Fig. 5. The flowchart of the collision avoidance system

For object detection, the machine learning algorithms are exploited. For image data obtained by the camera, object detection using the YOLOv3 algorithm [17] is implemented. In RSU, object detection is carried out by SSD-MobileNet [18] which is a popular network architecture for real-time object detection on embedded devices. While data about the surroundings are collected via two routes, in-vehicle sensors and V2I communication, the vehicle analyses the composite data conservatively. That is, if an object is detected by either the in-vehicle sensors or the V2I, the vehicle considers this as a potential collision. Then, in order to control the driving speed, it calculates the distance to the detected target. The

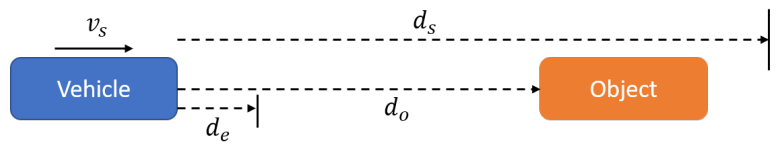

Fig. 6. Illustration for braking decision strategy

breaking strategy is depicted in Fig. $6 . d_{e}$ and $d_{s}$ represent as braking distance (min. distance in Fig. 5) and safety guaranteed distance, respectively. $d_{o}$ is the estimated distance between the vehicle and the detected object and $v_{s}$ represents the current speed, which is not faster than the maximum speed set by the driver. We only consider the case that $d_{o}$ is bigger than $d_{e}\left(d_{o}>d_{e}\right)$, and the vehicle will adjust the braking strategy according to the distance of $d_{o}-d_{e}$. For $d_{o}$ greater than $d_{s}$, the vehicle speed is maintained at $100 \%$ of the maximum speed set by the driver since the vehicle has enough distance to adjust its speed. If $d_{o}$ is grater than $d_{e}$ and less than $d_{s}$, the vehicle's brake is controlled appropriately so that the vehicle's speed can be maintained at $\left(d_{s}-d_{e}\right) /\left(d_{o}-d_{e}\right) \times v_{s}$.

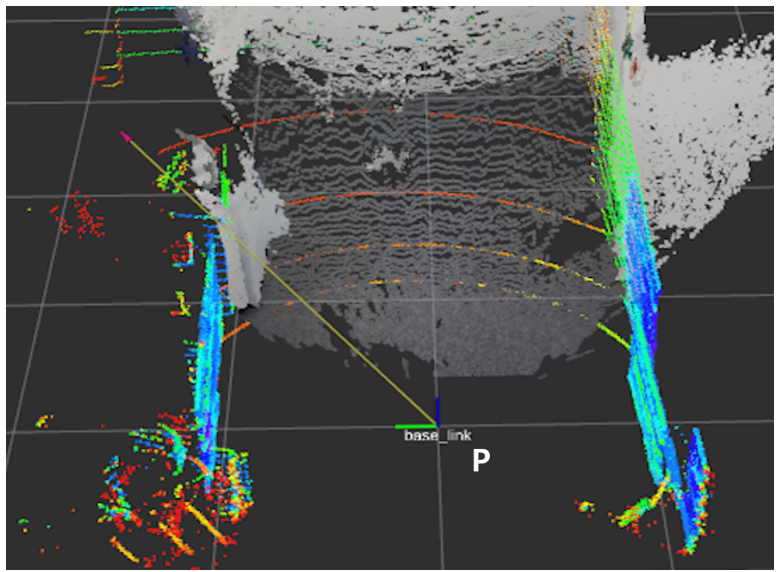

Fig. 7. Analysis of collected data

In this work, the software runs on Robot Operating System (ROS) [19], [20], a component-based middleware framework developed for large-scale integrative robotics research. The point cloud data [21] acquired from the LiDAR and the stereo cameras are monitored in real-time using RViz visualization provided in ROS as shown in Fig. 7.

Information including the current vehicle status, monitored image, the collision avoidance warning and the required vehicle control will be shown to the driver. Fig. 8 shows the GUI for the driver. The vehicle status including wheel speed, steering angle, battery information are shown in the vehicle Dashboard part. In box A, the image monitored by the in-vehicle camera is displayed and updated on a real-time basis. When static or moving obstacles are detected, a warning image will be displayed in box $\mathrm{C}$ and the vehicle reduces its speed gradually or abruptly depending on the distance between vehicle and obstacle. The change of speed is indicated in box B. There is an emergency brake in box $U$ in case that the driver needs to stop the vehicle for any reasons. 


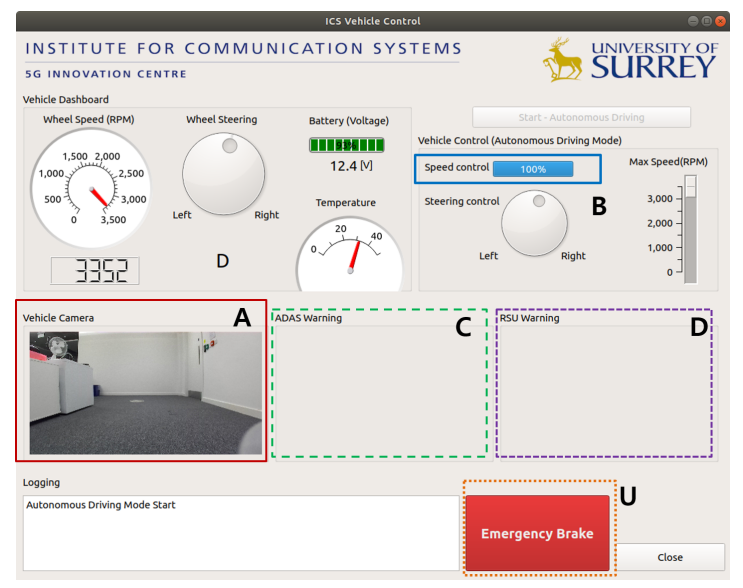

Fig. 8. The GUI to be displayed for the driver

\section{EXPERIMENTS}

The experimental scenarios to test our system are described and the functional operation of the collision avoidance system has been validated through tests.

(a)

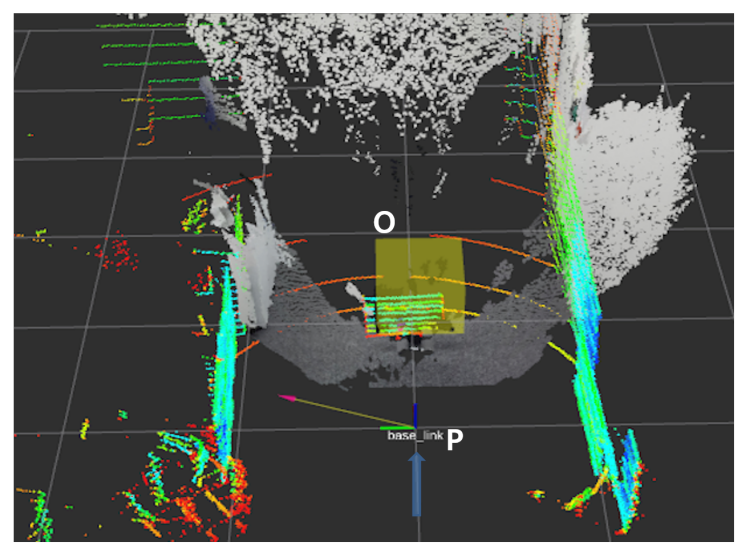

(b)

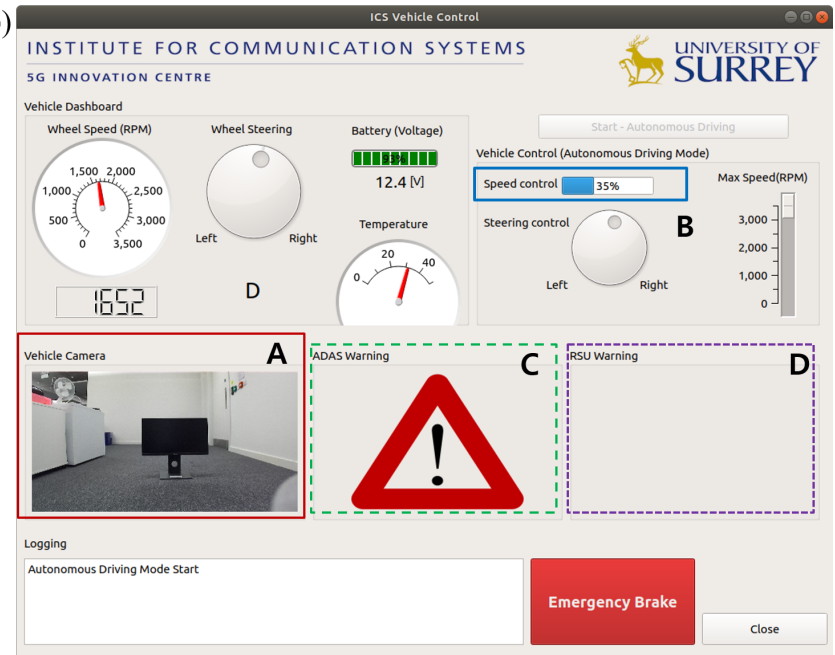

Fig. 9. Collision detection by in-vehicle sensors

\section{A. Experimental Scenarios and Performance Evaluation}

To validate the functional performance of collision detection, two scenarios are considered: 1) obstacles are present in the LOS area of the host vehicle, and 2) obstacles are present in the NLOS area.

1) Obstacles are present in the LOS area: This scenario is to show the effectiveness of data fusion using collected information from in-vehicle sensors. To focus on analysing in-vehicle's sensor data, the RSU is intentionally deactivated. It is considered that there is an object which can interfere with the vehicle's driving in the vehicle's route. Fig. 9 illustrates this scenario. While the vehicle is moving in direction of the blue arrow, the deployed sensors gather data in real-time. The collected data is analysed aiming to detect objects which may become or are obstacles to the vehicle. For this scenario, the analysed data is shown in Fig. 9(a). The physical distance of grid lines is $1 \mathrm{~m}$ and there are the vehicle at $\mathrm{P}$ and the object at $\mathrm{O}$. The vehicle at $\mathrm{P}$ can successfully detect the object at $\mathrm{O}$ and calculate the distance between $\mathrm{P}$ and $\mathrm{O}$. After detection of the object, the "possible collision" warning sign is displayed in the GUI (C in Fig. 9(b)) and the vehicle speed reduces to avoid collision (B in Fig. 9(b)). Although static objects are considered in this scenario, our system can detect moving

(a)

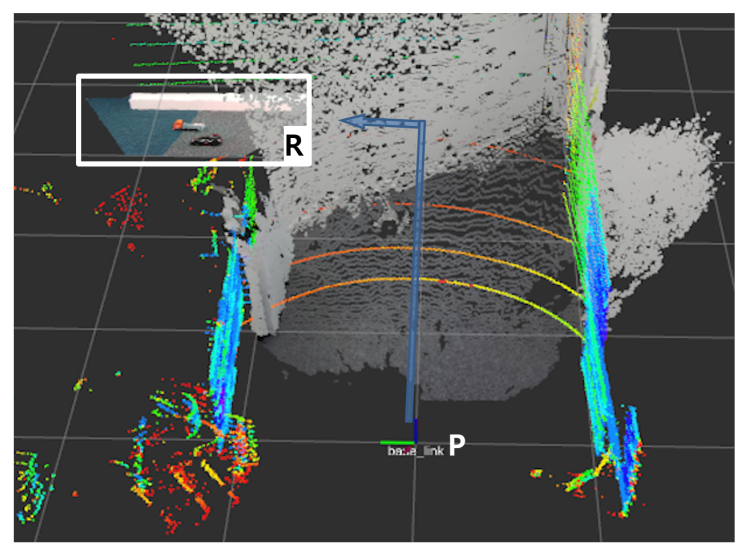

(b)

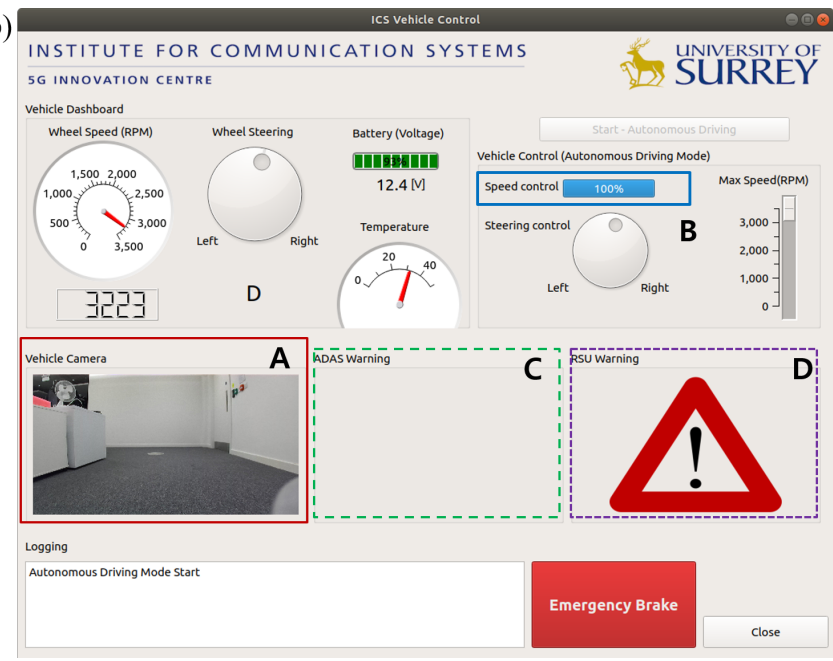

Fig. 10. Collision detection by the aid of VI2 communication 
objects and calculate varying distances to the object.

2) Obstacles are existed in the NLOS area: The effectiveness of V2I communication is tested in this scenario. The vehicle is supposed to move straight and make left turn at point $\mathrm{R}$ as the blue arrow shows. The RSU (e.g., traffic monitor camera) located at point $\mathrm{R}$ monitors the surrounding area. We intentionally put two static objects in the area indicated with box R in Fig. 10(a) while no objects are located in the straight route which can be monitored by in-vehicle sensors. In this case, sensors of vehicle at $\mathrm{P}$ are not detected anything in its LOS area and no warning sign is displayed in $\mathrm{C}$ Fig. 10(b). However, from the RSU at R, the warning message can be sent to the vehicle about the detected obstacles. By using obstacle's location information included in the warning message, the vehicle analyses the possibility of a collision and makes decision on its operation. More specifically, the vehicle judges whether the object is located in its driving route. In case that detected objects can interfere with the vehicle's route, the vehicle calculates the distance to the obstacles and also controls vehicle speed considering its current speed and location. In the considered scenario, while the vehicle finally identified obstacles more than $3 \mathrm{~m}$ away, it decides to alert the driver with the warning sign as shown in D in Fig. 10(b) but not to change the speed control at the moment ( $\mathrm{B}$ in Fig. 10(b)). As shown in this case, V2I communication covers the limitation of in-vehicle sensors (i.e., detection in NLOS area).

\section{Conclusion And Discussion}

It is envisaged that vehicles will in the future be highly connected with the aid of wireless networks to provide unprecedented driving experiences. They will offer a series of farreaching benefits such as significantly improved road safety, less traffic congestion, and reduced emission. In this context, while 5G/B5G will drive new connected vehicle services, it will also foster a shift towards a cloud-based vehicle and roadside infrastructure architecture.

Considering the clear trend towards vehicular communication and in-vehicle centralisation, this paper presents the collision avoidance system to assist safety driving based on in-vehicle data fusion with the aid of wireless networks. For the collision avoidance system, we use an open-source and the $1 / 10$ scale autonomous vehicle testbed. The system uses a modular hardware and software design enabling researches to shape and use the platform to fit their purpose. While the hardware dependent parts in software are implemented and managed as separate modules, the testbed allows easy adoption of algorithms tested in this testbed to other hardware systems (i.e., 1/5 scale vehicle). To operate the function of collision avoidance in our system, in-vehicle sensors including LiDAR, camera and IMU are exploited as well as V2I communication. The functional operation of our collision avoidance system is validated in scenarios where obstacles are present in the LOS and NLOS areas of the vehicles to show the effectiveness of in-vehicle sensors and V2I communication.
In future research, we will enhance the data fusion algorithms including comparison with reference algorithms developed in literature and additional features such as lane detection will be implemented and integrated to support more enhanced autonomous driving levels. Moreover, we will consider the scenario including vehicle-to-vehicle communications among multiple vehicles to effectively prevent incidents of both forward collision and rear-end collision.

\section{ACKNOWLEDGMENT}

We would like to acknowledge the support of 5GIC at the University of Surrey (http://www.surrey.ac.uk/5gic).

\section{REFERENCES}

[1] IEEE, "IEEE 5G and Beyond Technology Roadmap White Paper," IEEE Int'l Netwwork Generations Roadmap (INGR), Oct. 2017.

[2] Clear5G EU project, "Deliverable 1.1: System Specifications and Business Perspectives," Feb. 2019. [Online]. Available: https://clear5g.eu/sites/default/files/Clear5G_Deliverable_D1.1_Final.pdf

[3] 5G-HEART EU project, "Deliverable 2.1: Network Architecture and Slice Definition," Dec. 2019. [Online]. Available: https://5gheart.org/wpcontent/uploads/5G-HEART_D2.1.pdf

[4] X. Song and M. Yuan, "Performance Analysis of One-Way Highway Vehicular Networks With Dynamic Multiplexing of eMBB and URLLC Traffics," IEEE Access, vol. 7, pp. 118 020-118 029, Aug. 2019.

[5] C. R. Storck and F. Duarte-Figueiredo, "A Survey of 5G Technology Evolution, Standards, and Infrastructure Associated With Vehicle-toEverything Communications by Internet of Vehicles," IEEE Access, vol. 8, pp. $117593-117614$, June 2020.

[6] J. Ziegler, P. Bender, M. Schreiber, H. Lategahn, T. Strauss, C. Stiller, T. Dang, U. Franke, N. Appenrodt, C. G. Keller, E. Kaus, and et al., "Making Bertha Drive-An Autonomous Journey on a Historic Route," IEEE Intell. Transp. Syst. Mag., vol. 6, no. 2, pp. 8-20, 2014.

[7] A. Broggi et al., "PROUD-Public Road Urban Driverless-Car Test," IEEE Trans. Intell. Transp. Syst., vol. 16, no. 6, pp. 3508-3519, Dec. 2015.

[8] M. Baek, D. Jeong, D. Choi, and S. Lee, "Vehicle Trajectory Prediction and Collision Warning via Fusion of Multisensors and Wireless Vehicular Communications," Sensors, vol. 20, no. 1, Jan. 2020.

[9] Keysight Technologies, "How 5G Will Influence Autonomous Driving Vehicles," White Paper, July 2018.

[10] F. de Ponte Müller, E. M. Diaz, and I. Rashdan, "Cooperative positioning and radar sensor fusion for relative localization of vehicles," in Proc. 2016 IEEE Intell. Veh. Symp., Aug. 2016.

[11] 5G Americas, "Cellular V2X Communications Towards 5G," White Paper, Mar. 2018.

[12] F. Yang, J. Li, T. Lei, and S. Wang, "Architecture and key technologies for Internet of Vehicles: a survey," J. Commun. Info. Netw., vol. 2, June 2017.

[13] F. A. Teixeira et al., "Vehicular networks using the IEEE $802.11 \mathrm{p}$ standard: An experimental analysis," Vehicular Communications, vol. 1, no. 2, pp. 91-96, April 2014.

[14] G. Naik, B. Choudhury, and J. Park, "IEEE 802.11bd 5G NR V2X: Evolution of Radio Access Technologies for V2X Communications," IEEE Access, vol. 7, pp. 70 169-70 184, May 2019.

[15] F. Arena and G. Pau, "An Overview of Vehicular Communications," Future Internet, vol. 11, no. 2, Jan. 2019.

[16] D. Soudbakhsh and A. Eskandarian, Vehicle Lateral and Steering Control. London: Springer London, 2012.

[17] J. Redmon and A. Farhadi, "Yolov3: An incremental improvement," CoRR, vol. abs/1804.02767, 2018. [Online]. Available: http://arxiv.org/abs/1804.02767

[18] W. Liu, D. Anguelov, D. Erhan, C. Szegedy, S. E. Reed, C. Fu, and A. C. Berg, "SSD: single shot multibox detector," CoRR, vol. abs/1512.02325, 2015. [Online]. Available: http://arxiv.org/abs/1512.02325

[19] M. Quigley et al., "ROS: an open-source Robot Operating System," vol. 3, Jan. 2009.

[20] Robot Operating System. [Online]. Available: https://www.ros.org/

[21] Point Cloud Library. [Online]. Available: http://pointclouds.org 